\title{
The Role of Minimally Invasive Techniques in Scoliosis Correction Surgery
}

\author{
Michael B. Cloney ${ }^{(D)},{ }^{1}$ Jack A. Goergen, ${ }^{2}$ Angela M. Bohnen, ${ }^{1}$ Zachary A. Smith, \\ Tyler Koski, ${ }^{1}$ and Nader Dahdaleh ${ }^{1}$
}

${ }^{1}$ Department of Neurological Surgery, Feinberg School of Medicine, Chicago, IL, USA

${ }^{2}$ Feinberg School of Medicine, Chicago, IL, USA

Correspondence should be addressed to Michael B. Cloney; michael.cloney@yahoo.com

Received 28 August 2017; Accepted 11 December 2017; Published 24 January 2018

Academic Editor: Stephen Kavic

Copyright ( 2018 Michael B. Cloney et al. This is an open access article distributed under the Creative Commons Attribution License, which permits unrestricted use, distribution, and reproduction in any medium, provided the original work is properly cited.

\begin{abstract}
Objective. Recently, minimally invasive surgery (MIS) has been included among the treatment modalities for scoliosis. However, literature comparing MIS to open surgery for scoliosis correction is limited. The objective of this study was to compare outcomes for scoliosis correction patients undergoing MIS versus open approach. Methods. We retrospectively collected data on demographics, procedure characteristics, and outcomes for 207 consecutive scoliosis correction surgeries at our institution between 2009 and 2015. Results. MIS patients had lower number of levels fused $(p<0.0001)$, shorter surgeries $(p=0.0023)$, and shorter overall lengths of stay $(p<0.0001)$, were less likely to be admitted to the ICU $(p<0.0001)$, and had shorter ICU stays $(p=0.0015)$. On multivariable regression, number of levels fused predicted selection for MIS procedure $(p=0.004)$, and multiple other variables showed trends toward significance. Age predicted ICU admission and VTE. BMI predicted any VTE, and DVT specifically. Comorbid disease burden predicted readmission, need for transfusion, and ICU admission. Number of levels fused predicted prolonged surgery, need for transfusion, and ICU admission. Conclusions. Patients undergoing MIS correction had shorter surgeries, shorter lengths of stay, and shorter and fewer ICU stays, but there was a significant selection effect. Accounting for other variables, MIS did not independently predict any of the outcomes.
\end{abstract}

\section{Introduction}

Adult scoliosis is a spinal deformity typically caused by asymmetrical disc degeneration, osteoporosis, and vertebral body compression fractures [1]. When nonsurgical treatment fails, there are multiple surgical techniques that can be used [2]. The goals of surgery are to improve functionality, relieve pain, improve cosmesis, and prevent curve progression [3]. Whether performed posteriorly or anteriorly, open techniques are associated with large blood loss, muscle injury and denervation, significant postoperative pain, and other complications $[4,5]$.

Minimally invasive surgery (MIS) potentially avoids or lessens these complications due to its ability to reduce intraoperative blood loss, soft tissue damage, infection, postoperative pain, and recovery time [6]. The safety and feasibility of MIS for adult degenerative scoliosis have already been established [7]. Also, results have previously been reported that showed similar clinical improvement for patients who underwent open surgery versus MIS [8]. Furthermore, the patients who underwent MIS had lower morbidity and complication rates and significantly shorter hospital stays [8].

While these initial results are promising, these studies were on small subsets of patients with many confounding variables. The literature comparing open surgery versus MIS for scoliosis correction is limited; therefore, the need exists for further investigation to determine the efficacy of MIS. Here, we compared MIS and open scoliosis surgery with respect to selection for surgical technique and outcomes, including readmission rates, reoperation rates, bleeding, and clotting complications. 
TABLE 1: Approaches and techniques used in minimally invasive operations.

\begin{tabular}{lc}
\hline & Number of operations using technique \\
\hline Anterior approach & 2 \\
Posterior approach & 11 \\
Lateral approach & 1 \\
Percutaneous screws & 9 \\
Interbody fusion & 10 \\
\hline
\end{tabular}

\section{Methods}

2.1. Patient Population. Patients were identified using the Northwestern University Electronic Data Warehouse (EDW). The EDW is an institution-specific registry clinical data repository jointly funded by Northwestern Memorial Hospital (NMH), Northwestern Medical Faculty Foundation (NMFF), and Northwestern University Feinberg School of Medicine. We identified all patients who underwent surgery for scoliosis in the Departments of Neurological Surgery or Orthopedic Surgery at Northwestern University between January 1, 2009, and May 31, 2015, as determined by the preoperative indication for surgery provided by the surgeon.

2.2. Clinical and Demographic Data. Data on patients' age, sex, race, BMI, smoking status (ever smoker versus never smoker), number of comorbid diagnoses, and insurance type (private versus other, including Medicare, Medicaid, and disability insurance) at the time of presentation were retrospectively collected for analysis. Data were also collected on the number of levels fused and length of surgery, as well as whether the patients' scoliosis correction involved a staged procedure, interbody fusion, laminectomy, or osteotomy. Data on surgical techniques and approaches used were also collected. The approaches used included lateral, posterior, and anterior. Of the 14 minimally invasive operations, most utilized an interbody fusion with percutaneous screws; see Table 1 for details.

2.3. Outcome Measures. Information about complications within 30 days after the surgery included the cumulative 30day incidence and timing of VTEs (defined as either DVT or $\mathrm{PE}$ ), all-cause readmissions, reoperations, ICU admission, length of ICU stay, length of hospital stay, and incidence of death.

2.4. Statistical Methods. Microsoft Excel 2011 (Microsoft, Redmond, WA, USA) was used to manage data. Statistical analysis was performed using Stata 12.0 (StataCorp, College Station, TX, USA) and Prism 6.0b (GraphPad Software, Inc., La Jolla, CA, USA). Parametric data was given as mean \pm standard deviation and compared using a $t$-test. Nonparametric data was compared using Mann-Whitney $U$ test or Chi-square test, as appropriate. Regression analysis was performed using stepwise logistic regression, with an inclusion threshold for the multivariable model of $p<0.10$

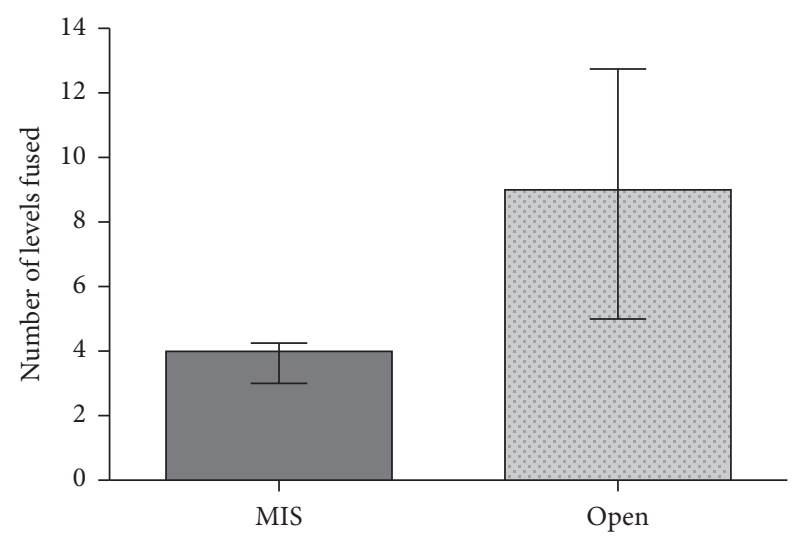

FIGURE 1: Number of levels fused for patients undergoing MIS approach versus open approach.

for candidate variables on single-variable logistic regression. A value of $p<0.05$ was considered statistically significant.

\section{Results}

3.1. Demographic Characteristics. There was no difference in age $(64.5 \pm 1.4$ versus $58.1 \pm 1.2, \Delta-6.44[-15.11,2.22], p=$ $0.1442)$, gender ( $35.7 \%$ male versus $25.9 \%$ male, 1.588889 $[0.5081890,4.967774], p=0.5304)$, race $(p=0.3243)$, insurance type $(p=0.6694)$, smoking status $(p=0.4284)$, BMI $(26.1 \pm 0.6$ versus $26.8 \pm 0.4, \Delta 0.72 \pm 1.47[-2.17,3.61]$, $p=0.6242)$, or comorbid disease burden $(p=0.4499)$. On multivariable regression, age (OR 1.076323 [0.9977851, 1.161042], $p=0.057$ ) and having private insurance (OR $3.735077[0.9058839,15.40021], p=0.068)$ showed trends toward selection for MIS surgery (see Table 2).

3.2. Procedure Data. MIS patients were equally likely to have staged surgery (OR 0.6507177 [0.1749019, 2.420978], $p=0.5186$ ), decompression (OR 0.3597285 [0.04548798, 2.844809], $p=0.3127$ ), osteotomy (OR 0.2604895 $[0.03313165,2.048035], p=0.1703)$, and allograft (OR $0.6491885[0.2098068,2.008732], p=0.4505)$. There was a trend toward significance in surgical approach $(p=0.0857)$ and surgery involving the thoracic spine (OR 0.1539582 [0.008965574, 2.643795], $p=0.0805)$. MIS patients were less likely to have autograft (OR 0.1382386 [0.01769585, 1.079909], $p=0.0289$ ) and had a lower number of levels fused (4.0 versus 9.0, $\Delta 5.0[2.0,7.0], p<0.0001$, Figure 1). There was significantly more variance in the number of levels fused among patients undergoing open surgery $(p<$ $0.0001)$. On multivariable regression, the number of levels fused predicted selection for MIS procedure (OR 0.6079009 [0.4340611,0.8513629], $p=0.004)$, and there was a trend toward significance for selection for MIS among patients undergoing a posterior approach (OR 3.43426 [0.8365153, 14.09913], $p=0.087$ ) and not requiring surgical decompression (OR 0.1319887 [0.0147237, 1.183196], $p=0.070$ ) (see Table 3). 
TABLE 2: Patient demographic data for MIS approach versus open approach.

\begin{tabular}{|c|c|c|c|}
\hline & MIS patients & Open patients & $p$ value \\
\hline Age & 64.5 years (mean) & 58.1 years (mean) & 0.1442 \\
\hline Gender & $35.7 \%$ male & $25.9 \%$ male & 0.5304 \\
\hline Race & $100 \%$ Caucasian & $\begin{array}{c}\text { 86.01\% Caucasian, } 4.15 \% \text { African } \\
\text { American, } 9.84 \% \text { other }\end{array}$ & 0.3243 \\
\hline Insurance type & 10 private, 4 Medicare & $\begin{array}{c}108 \text { private, } 77 \text { Medicare, } 7 \text { Medicaid, } 1 \\
\text { other }\end{array}$ & 0.6694 \\
\hline Smoking status & $\begin{array}{c}11 \text { never smoked, } 3 \text { quit more than } 12 \\
\text { months ago }\end{array}$ & $\begin{array}{l}119 \text { never smoked, } 53 \text { quit more than } 12 \\
\text { months ago, } 21 \text { current smokers or } \\
\text { smoked within last } 12 \text { months }\end{array}$ & 0.4284 \\
\hline BMI & 26.1 (mean) & $26.8($ mean $)$ & 0.6242 \\
\hline Comorbidities & $\begin{array}{c}2.43 \text { comorbidities per patient } \\
\text { (cardiac, renal, pulmonary, endocrine, } \\
\text { or hypertension) }\end{array}$ & $\begin{array}{l}2.8 \text { comorbidities per patient (cardiac, } \\
\text { renal, pulmonary, endocrine, or } \\
\text { hypertension) }\end{array}$ & 0.4499 \\
\hline
\end{tabular}

TABLE 3: Surgical procedure data for MIS approach versus open approach.

\begin{tabular}{lccc}
\hline & Odds ratio for MIS patients/open approach patients & Confidence interval & $p$ value \\
\hline Posterior approach & 3.43426 & {$[0.8365153,14.09913]$} & 0.087 \\
Number of levels fused & 0.6079009 & {$[0.4340611,0.8513629]$} & 0.004 \\
Staged procedure & 0.6507177 & {$[0.1749019,2.420978]$} & 0.5186 \\
Osteotomy & 0.2604895 & {$[0.03313165,2.048035]$} & 0.1703 \\
Decompression & 0.3597285 & {$[0.04548798,2.844809]$} & 0.3127 \\
Allograft & 0.6491885 & {$[0.2098068,2.008732]$} & 0.4505 \\
Autograft & 0.1382386 & {$[0.01769585,1.079909]$} & 0.0289 \\
Operation involving Thoracic spine & 0.1539582 & {$[0.008965574,2.643795]$} & 0.0805 \\
\hline
\end{tabular}

3.3. Outcomes for MIS versus Open Scoliosis Correction. MIS surgery was significantly shorter (287.0 minutes versus 433.0 minutes, HR 2.319 [1.604, 8.342], $p=0.0023$, Figure 2) and was less likely to last $\geq 6$ hours (OR 0.2280405 [0.0751441, 0.6920369], $p=0.0051)$. MIS patients had shorter overall lengths of stay (4.5 days versus 8.0 days, HR 3.032 [3.725, 22.61], $p<0.0001$, Figure 3), were less likely to be admitted to the ICU (OR 0.08779576 [0.02348702, 0.3281854], $p<$ $0.0001)$, and had shorter ICU stays (19.0 hours versus 48.5 hours, HR 5.174 [5.200, 866.7], $p=0.0015)$.

On single-variable analysis, MIS patients were equally likely to experience the following within 30 days of surgery: readmission (OR 0.3271202 [0.01872778, 5.713847], $p=$ 0.2318 ), reoperation (OR 1.181818 [0.06218783, 22.45929], $p=1.0000)$, DVT (OR 0.3477832 [0.01986991, 6.087253], $p=0.2464), \mathrm{PE}(\mathrm{OR} 3.634615[0.3782204,34.92786], p=$ 0.2328 ), any VTE (OR 0.8509616 [0.1044311, 6.934095], $p=$ 0.8800 ), and postoperative death (OR 2.668966 [0.1222150, 58.28564], $p=0.7034)$. MIS patients were less likely to require transfusion (OR 0.1231231 [0.02681325, 0.5653661], $p=0.0017)$.

3.4. Predictors of Outcomes after Scoliosis Correction. On multivariable regression, BMI predicted DVT within 30 days postoperatively (OR 1.130749 [1.021063, 1.252219], $p=$ 0.018 ), and age (OR $1.048752[0.9975131,1.102623], p=$ 0.063 ) showed a trend toward significance. Number of levels

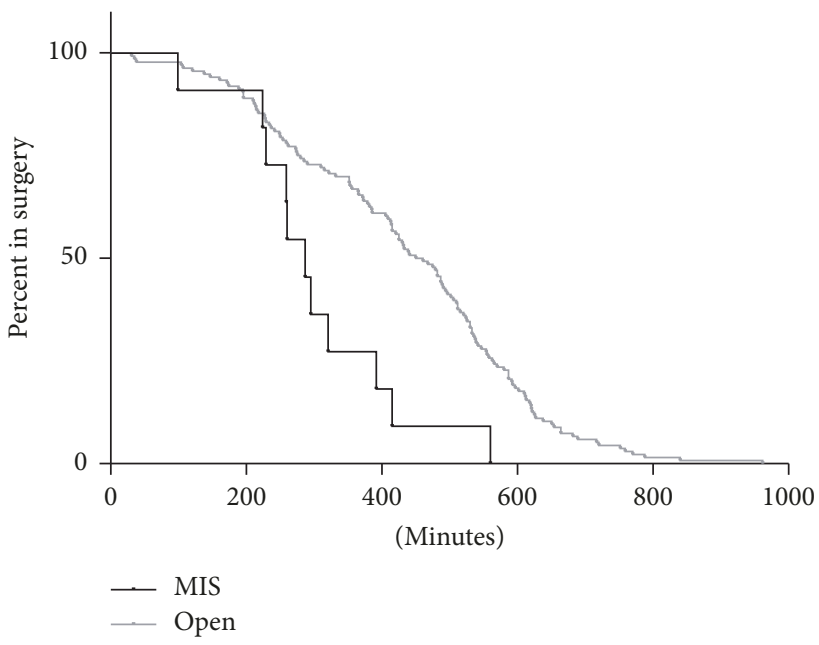

Figure 2: Comparison in surgery length between MIS and open approach patients.

fused showed a trend toward significance in predicting PE 30 days postop (OR $1.251054[0.9814253,1.594758], p=$ 0.071). Age (OR 1.053943 [1.000293, 1.11047], $p=0.049$ ) and BMI (OR 1.143371 [1.037006, 1.260645], $p=0.007$ ) predicted VTE within 30 days postop. Comorbid disease burden predicted readmission within 30 days postop (OR 


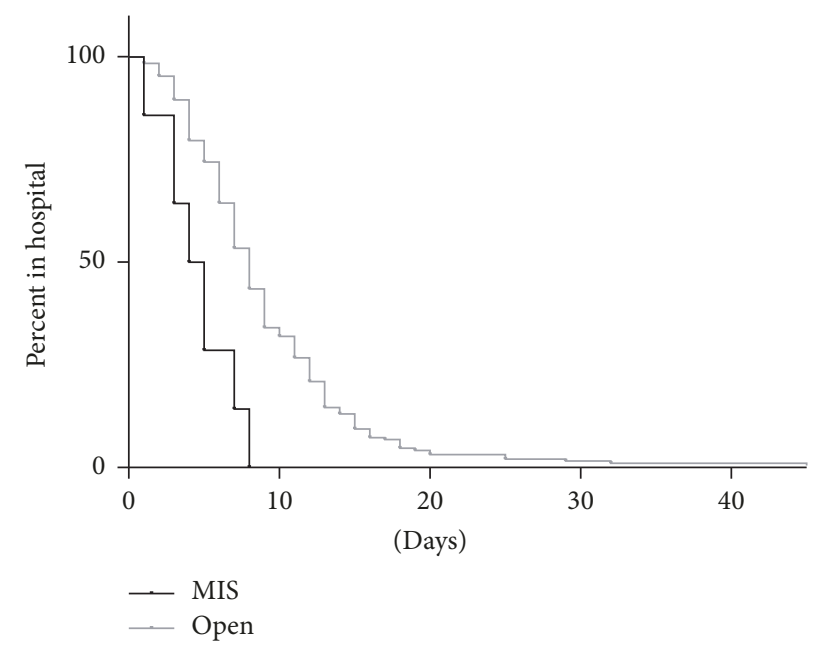

FIGURE 3: Comparison in LOS between MIS and open approach patients.

2.543268 [1.376737, 4.698219], $p=0.003$ ), and involvement of the thoracic spine showed a trend toward significance (OR 0.1084136 [0.0109545, 1.072934], $p=0.057$ ). Number of levels fused predicted prolonged surgery (surgery $>6 \mathrm{~h}$ ) (OR 1.452142 [1.233844, 1.709062], $p<0.001$ ), and a history of smoking showed a trend toward significance (OR 0.0040854 [7.33e - 06, 2.275654], $p=0.088)$. Number of levels fused (OR 1.297174 [1.182993, 1.422377], $p<$ 0.001 ) and comorbid disease burden (OR 1.297174 [1.182993, 1.422377], $p<0.001$ ) predicted the need for transfusion, and osteotomy showed a trend toward significance (OR 2.359625 $[0.9492208,5.865686], p=0.065)$. Age (OR 0.9512098 $[0.9089738,0.9954083], p=0.031)$, gender (OR 3.299076 $[1.06138,10.25448], p=0.039)$, comorbid disease burden (OR 1.686387 [1.123766, 2.530687], $p=0.012$ ), number of levels fused (OR 2.089387 [1.615015, 2.703095], $p<0.001$ ), and undergoing a staged procedure (OR 5.321398 [1.470397, 19.25826], $p=0.011$ ) all predicted ICU admission.

\section{Discussion}

Minimally invasive surgical techniques could potentially reduce the morbidity associated with traditional open surgical techniques in scoliosis correction [9-11]. Currently, the literature on MIS for scoliosis correction is limited. Many of the studies performed to date observed relatively few patients, and multiple systematic reviews have concluded that more research is needed $[9,12]$. Our study examined 207 scoliosis correction surgeries and identified selection factors for MIS, how MIS outcomes compare to open surgery outcomes, and predictors of outcomes for each technique.

Importantly, the median number of levels fused predicted selection for MIS technique with MIS patients having fewer levels fused (4 versus 9, $p<0.0001$ ). Many previous studies did not search for a selection effect for MIS versus open surgery selection. One meta-analysis on scoliosis correction, by Dangelmajer et al., did examine selection bias and found that both older patients and patients with less severe deformities were more likely to be selected for an MIS technique [13]. Our analysis agrees with this finding.

Furthermore, having private insurance $(p=0.068)$, undergoing a posterior approach $(p=0.087)$, and not requiring surgical decompression $(p=0.070)$ each showed a trend toward selection for MIS. Similarly to our finding that private insurance was an important determinant of surgery choice, a study by Park and Ha revealed that the cost of the MIS technique determined which patients underwent MIS versus open technique [6]. Another group separated the patients by allocating the private hospital patients to receive MIS and the public hospital patients in their study to receive the open technique [14]. This trend of selection biases between the two groups was consistent across most of the studies that reported patient selection information, limiting the ability to conclude a true difference in outcomes between the MIS and open techniques.

In our study, there was significantly more variance in the number of levels fused among patients undergoing open surgery $(p<0.0001)$. Dangelmajer et al. came to the same conclusion in their systematic review and attributed it to the fact that patients undergoing open procedure had a larger preoperative scoliosis [13]. This result shows that an open technique can be used for a broader range of spinal levels than MIS.

MIS surgery was significantly shorter (287.0 minutes versus 433.0 minutes, $p=0.0023$, Figure 2 ) and patients undergoing MIS were less likely to have surgery last $>6$ hours $(p=0.0051)$ based on single-variable analysis. Anand et al. noted that their surgical outcomes data for MIS scoliosis correction was similar to open correction outcomes data when compared to the literature [8]. However, a metaanalysis on MIS versus open approach in degenerative lumbar disease revealed significant variability in operating times [15]. For example, one study found an average operating time of 161 minutes for the MIS approach compared to 375 minutes for the open approach [16]. In contrast, a study in the same meta-analysis found an average operating time of 159.2 minutes for the MIS approach versus 113.06 minutes for the open approach [17]. We suspect that confounding variables have an important impact on operating time, which would explain the significant variability between studies.

MIS patients also had shorter overall lengths of stay based on single-variable analysis ( 4.5 days versus 8.0 days, $p<$ 0.0001 , Figure 3 ). While it seems promising that MIS patients typically had a shorter length of stay, the results from other studies are variable, potentially indicating a selection effect $[6,14,18]$. Our length of stay results were consistent with a meta-analysis by Phan et al. that found a median length of stay of 4.7 days for the MIS approach and 8 days for the open approach [15]. Although our results are consistent with the meta-analysis, the presence of confounding variables makes it uncertain if MIS versus open surgery is the etiology of the variability in length of stay.

Compared to patients undergoing open surgery, MIS patients were less likely to be admitted to the ICU ( $p<$ 0.0001 ) but did have shorter ICU stays when it was required 
(19.0 hours versus 48.5 hours, $p=0.0015)$. These results are consistent with previous groups who found that open surgery patients typically had more complications following surgical treatment $[13,19]$. However, similarly to our study, these groups also noted the presence of confounding variables such as age and preoperation severity of deformity that could have attributed to these results. In fact, in our analysis, open surgery did not predict ICU admission on multivariable regression.

While MIS or open correction was not independently associated with ICU admission, the number of levels fused did independently predict ICU admission $(p<0.001)$. As the number of levels fused was also independently associated with the selection of technique, it may be a confounding factor that accounts for the significant difference in likelihood of ICU admission on single-variable analysis. Similarly, age was likely a confounding factor, as age showed a trend toward significance in predicting technique, and was a significant predictor of ICU admission ( $p=0.031)$.

Our finding that comorbid disease burden independently predicted ICU admission $(p=0.012)$ and readmission within 30 days $(p=0.003)$ is consistent with the existing spine surgery literature $[20,21]$. Cardiac, GI, and respiratory issues that were present before the operation are frequent causes of ICU admission and readmission and appear to be an important factor when comparing MIS versus open technique for scoliosis correction as well.

On multivariable regression analysis, age $(p=0.049)$ and BMI $(p=0.007)$ predicted VTE within 30 days postop. These variables are typically found to be strongly associated with such outcomes in spinal surgery, as evidenced in numerous previous studies [22-24]. Importantly, MIS surgery was not found to be an independent predictor of any outcome analyzed during multivariable regression. So, although we found that the typical outcome predictors (age, BMI, and comorbid disease burden) were significant in this study, we did not find any significant difference in patient outcome based on MIS versus open technique alone.

Our study has a number of important limitations. The study was conducted retrospectively and is subject to the biases inherent to this study design. A prospective study would enable us to further understand if the trends we discovered (private insurance, number of levels fused showing selection for MIS) were actively affecting the surgeon's decision whether to use an MIS or open approach. The operations we collected data on varied in the minimally invasive technique and approach used, which made it a less homogenous population to draw conclusions from. As a single-institution study, it only reflects the clinical decision-making of our spine surgeons with respect to patient selection and management. Our series is limited by its size, and a larger series would allow for a more thorough comparison between MIS and open surgery. Our study does not provide radiographic comparisons of corrections, as is common in the scoliosis literature. However, multiple prior studies have compared radiographic outcome for MIS and open scoliosis correction, the results of which have been meta-analyzed [2, 5, 7, 13, 25-27]. Despite its limitations, our study contributes to the existing literature on scoliosis correction by examining selection factors for
MIS versus open surgery, as well as a variety of perioperative outcomes.

\section{Conclusion}

Patients undergoing MIS scoliosis correction had shorter surgeries, shorter lengths of stay, and shorter and fewer ICU stays, but there was a significant selection effect. Accounting for other clinical variables, undergoing MIS surgery did not independently predict any of the outcomes analyzed.

\section{Conflicts of Interest}

Dr. Koski is a consultant at NuVasive.

\section{References}

[1] M. Aebi, "The adult scoliosis," European Spine Journal, vol. 14, no. 10, pp. 925-948, 2005.

[2] S. Yadla, M. G. Maltenfort, J. K. Ratliff, and J. S. Harrop, "Adult scoliosis surgery outcomes: a systematic review," Neurosurg Focus, vol. 28, no. E3, 2010.

[3] N. Anand, E. M. Baron, and B. Khandehroo, "Is circumferential minimally invasive surgery effective in the treatment of moderate adult idiopathic scoliosis?" Clinical Orthopaedics and Related Research, vol. 472, pp. 1762-1768, 2014.

[4] J. J. Regan, H. Yuan, and P. C. McAfee, "Laparoscopic fusion of the lumbar spine: Minimally invasive spine surgery: A prospective multicenter study evaluating open and laparoscopic lumbar fusion," The Spine Journal, vol. 24, no. 4, pp. 402-411, 1999.

[5] M. Y. Wang and P. V. Mummaneni, "Minimally invasive surgery for thoracolumbar spinal deformity: initial clinical experience with clinical and radiographic outcomes," Neurosurg Focus, vol. 28, p. E9, 2010.

[6] Y. Park and J. W. Ha, "Comparison of one-level posterior lumbar interbody fusion performed with a minimally invasive approach or a traditional open approach," The Spine Journal, vol. 32, no. 5, pp. 537-543, 2007.

[7] E. Dakwar, R. F. Cardona, D. A. Smith, and J. S. Uribe, "Early outcomes and safety of the minimally invasive, lateral retroperitoneal transpsoas approach for adult degenerative scoliosis," Neurosurg Focus, vol. 28, no. 3, p. E8, 2010.

[8] N. Anand, R. Rosemann, B. Khalsa, and E. M. Baron, "Midterm to long-term clinical and functional outcomes of minimally invasive correction and fusion for adults with scoliosis," Neurosurg Focus, vol. 28, E6, 2010.

[9] K. Bach, A. Ahmadian, A. Deukmedjian, and J. S. Uribe, "Minimally invasive surgical techniques in adult degenerative spinal deformity: a systematic review," Clinical Orthopaedics and Related Research, vol. 472, pp. 1749-1761, 2014.

[10] E. M. Baron and T. J. Albert, "Medical complications of surgical treatment of adult spinal deformity and how to avoid them," Spine (Phila Pa 1976), vol. 31, pp. S106-118, 2006.

[11] S. S. Hu, "Blood loss in adult spinal surgery," European Spine Journal, vol. 13, no. S01, pp. S3-S5, 2004.

[12] N. Anand, E. M. Baron, and S. Kahwaty, "Evidence basis/ outcomes in minimally invasive spinal scoliosis surgery," Neurosurgery Clinics of North America, vol. 25, pp. 361-375, 2014. 
[13] S. Dangelmajer, P. L. Zadnik, S. T. Rodriguez, Z. L. Gokaslan, and D. M. Sciubba, "Minimally invasive spine surgery for adult degenerative lumbar scoliosis," Neurosurgical Focus, vol. 36, no. 5, p. E7, 2014.

[14] R. J. Mobbs, P. Sivabalan, and J. Li, "Minimally invasive surgery compared to open spinal fusion for the treatment of degenerative lumbar spine pathologies," Journal of Clinical Neuroscience, vol. 19, pp. 829-835, 2012.

[15] K. Phan, P. J. Rao, A. C. Kam, and R. J. Mobbs, "Minimally invasive versus open transforaminal lumbar interbody fusion for treatment of degenerative lumbar disease: systematic review and meta-analysis," European Spine Journal, vol. 24, pp. 10171030, 2015.

[16] W. A. R. Sulaiman and M. Singh, "Minimally invasive versus open transforaminal lumbar interbody fusion for degenerative spondylolisthesis grades 1-2: patient-reported clinical outcomes and cost-utility analysis," The Ochsner Journal, vol. 14, no. 1, pp. 32-37, 2014.

[17] N.-F. Tian and F.-M. Mao, "Minimally invasive versus open transforaminal lumbar interbody fusion: a meta-analysis based on the current evidence," European Spine Journal, vol. 23, no. 4, pp. 929-930, 2014.

[18] F. Shunwu, Z. Xing, Z. Fengdong, and F. Xiangqian, "Minimally invasive transforaminal lumbar interbody fusion for the treatment of degenerative lumbar diseases," The Spine Journal, vol. 35, no. 17, pp. 1615-1620, 2010.

[19] R. E. Isaacs, J. Hyde, J. A. Goodrich, W. B. Rodgers, and F. M. Phillips, "A prospective, nonrandomized, multicenter evaluation of extreme lateral interbody fusion for the treatment of adult degenerative scoliosis: perioperative outcomes and complications," The Spine Journal, vol. 35, pp. S322-330, 2010.

[20] H. F. Kay, S. Chotai, J. B. Wick, D. P. Stonko, M. J. McGirt, and C. J. Devin, "Preoperative and surgical factors associated with postoperative intensive care unit admission following operative treatment for degenerative lumbar spine disease," European Spine Journal, vol. 25, no. 3, pp. 843-849, 2016.

[21] R. A. McCormack, T. Hunter, N. Ramos, R. Michels, L. Hutzler, and J. A. Bosco, "An Analysis of Causes of Readmission After Spine Surgery," The Spine Journal, vol. 37, no. 14, pp. 1260-1266, 2012.

[22] M. P. Glotzbecker, C. M. Bono, K. B. Wood, and M. B. Harris, "Thromboembolic disease in spinal surgery: a systematic review," Spine (Phila Pa 1976), vol. 34, pp. 291-303, 2009.

[23] S. Z. Goldhaber and H. Bounameaux, "Pulmonary embolism and deep vein thrombosis," Lancet, vol. 379, pp. 1835-1846, 2012.

[24] K. Papadimitriou, A. G. Amin, R. M. Kretzer et al., "Thromboembolic events and spinal surgery," Journal of Clinical Neuroscience, vol. 19, no. 12, pp. 1617-1621, 2012.

[25] S. H. Berven, V. Deviren, J. A. Smith, S. H. Hu, and D. S. Bradford, "Management of fixed sagittal plane deformity: outcome of combined anterior and posterior surgery," Spine (Phila Pa 1976), vol. 28, no. 15, pp. 1710-1715, 2003.

[26] M. O. Kelleher, M. Timlin, O. Persaud, and Y. R. Rampersaud, "Success and failure of minimally invasive decompression for focal lumbar spinal stenosis in patients with and without deformity," The Spine Journal, vol. 35, no. 19, pp. E981-E987, 2010.

[27] W. Liu, X. S. Chen, L. S. Jia, and D. W. Song, "The clinical features and surgical treatment of degenerative lumbar scoliosis: a review of 112 patients," Orthopaedic Surgery, vol. 1, pp. 176-183, 2009. 


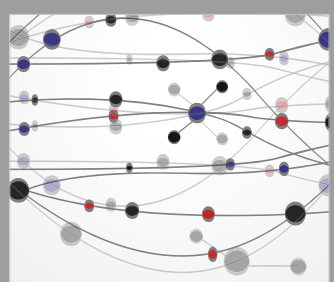

The Scientific World Journal
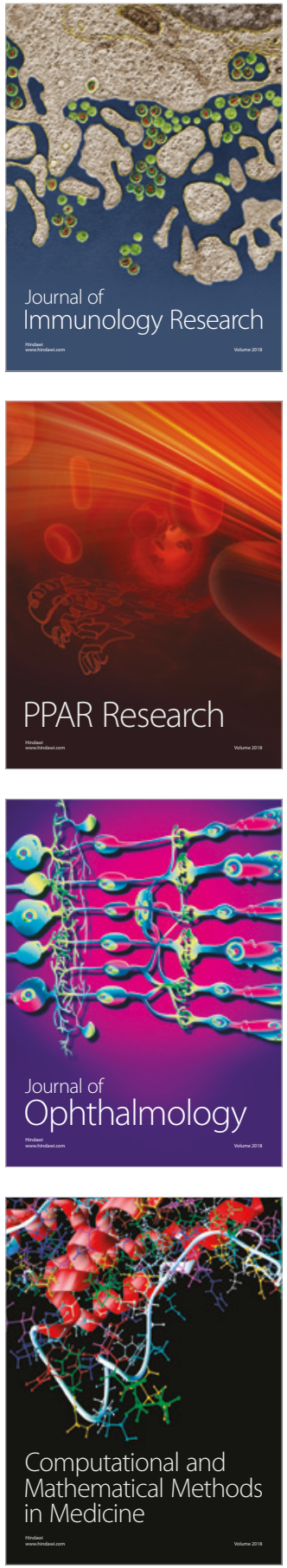

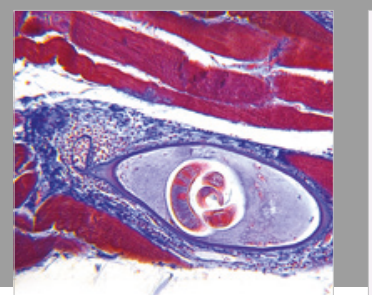

Gastroenterology Research and Practice

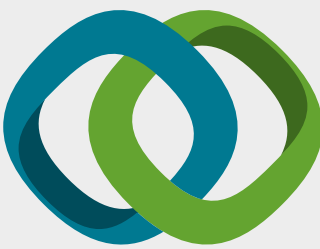

\section{Hindawi}

Submit your manuscripts at

www.hindawi.com
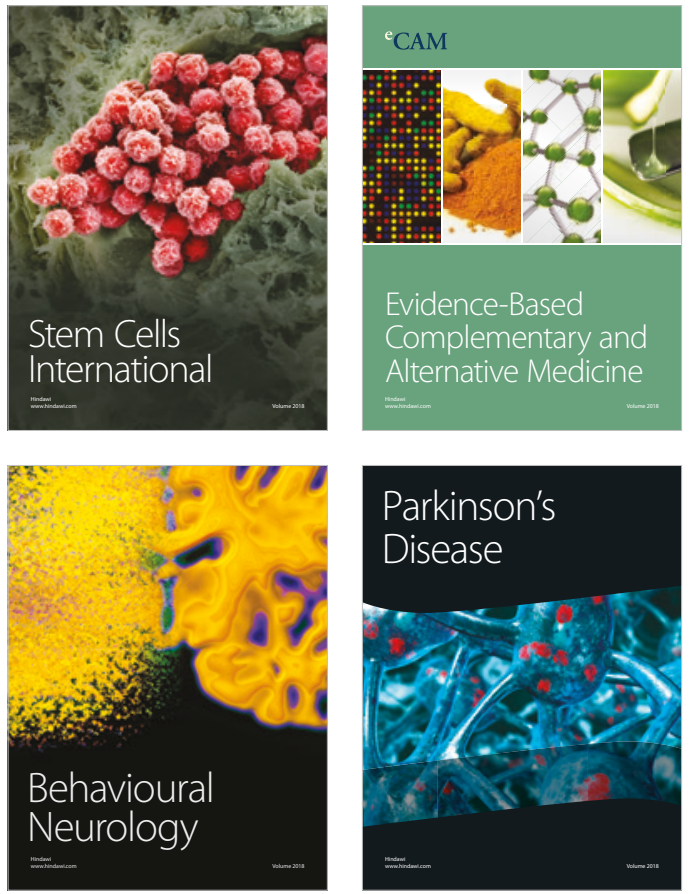

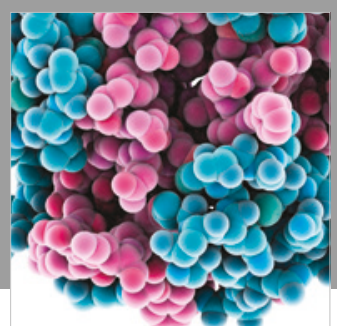

ournal of

Diabetes Research

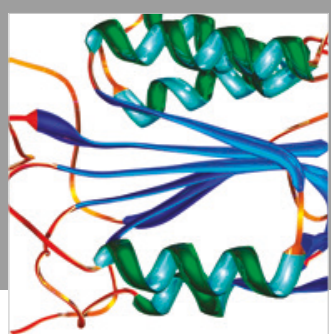

Disease Markers
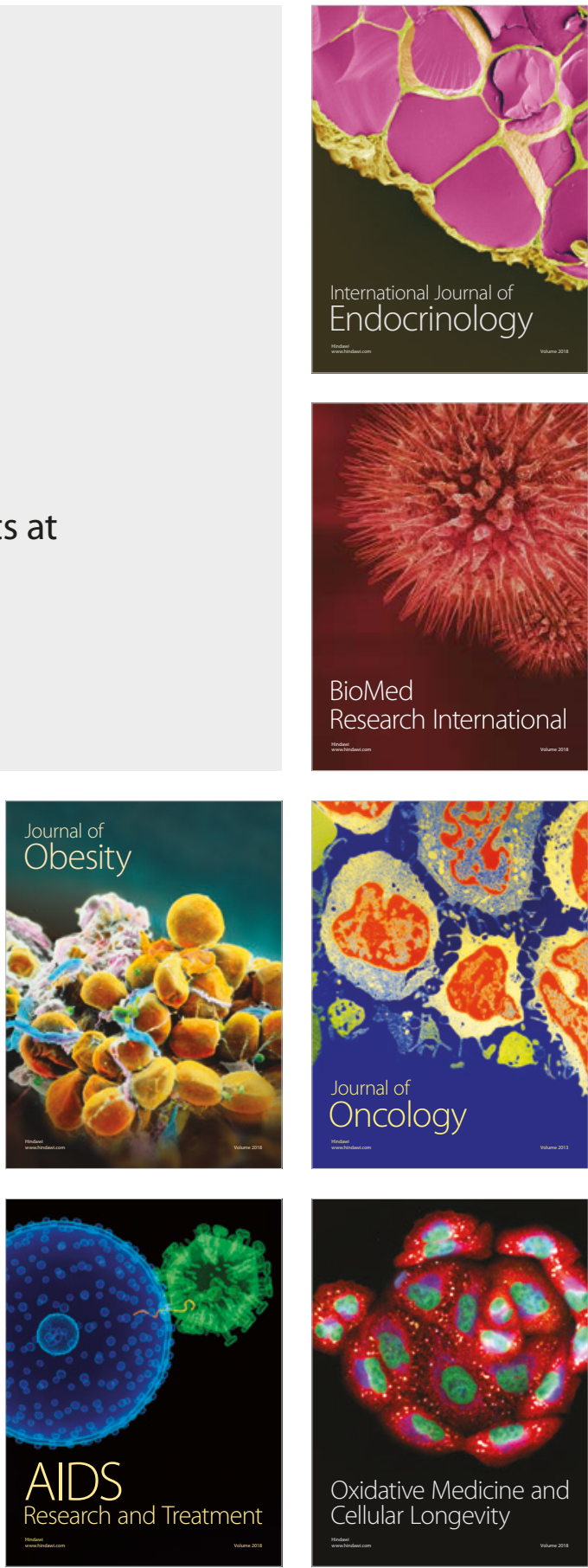